Okajimas Fol. anat. jap., $54: 195-204,1977$

\title{
A Scanning Electron Microscopic Study of the Splenic Sheathed Vessel of the Pig
}

By

\author{
Yoshikuni Ohta, Takao Tokioka, Hitoshi Okuda \\ and Fumihiko Suwa
}

Department of Anatomy, Osaka Dental University, Osaka 540, Japan

(with 9 figures)

-Received for Publication, March 23, 1977-

The morphology and function of the splenic sheath have been thoroughly discussed. Schweigger-Seidel (1863) first pointed out that a high permeability was seen on the wall of the sheathed vessel. Later some research workers who used color suspension materials for investigating the sheathed vessel have mentioned that the material leaked from the wall of the vessel into the sheath. Ohta (1957) described that the injected acryl plastic leaked from some spots of the sheathed capillary wall and displayed a definite route within the sheath under the light microscope.

This paper will deal with the passageway within the sheath under the scanning electron microscope as a further study on his findings.

\section{Materials and Methods}

Fresh spleens of pigs were washed with Ringer's solution through the splenic artery and fixed by perfusion of the glutaraldehyde. The plastic was injected into the splenic artery only or into the splenic artery and vein, of both fresh and fixed spleens by means of the acryl plastic injection method (Taniguchi et al. 1952 and 1955). The injected spleen was prepared into the corrosion specimen of the splenic vascular system by digesting soft structures with sodium hydroxide. The specimen treated with the ultrasonic washing appliance was microdissected under the stereoscopic dissecting microscope to pick up the cast of a sheath or a sheath with the surrounding red pulp and splenic sinuses. Then these portions were cut under the freezing microtome in both longitudinal and transverse directions for scanning electron microscopy.

The spleen washed out with Ringer's solution was perfused with 
formaldehyde-glutaraldehyde in $0.2 \mathrm{M}$ sodium cacodilate buffer, $\mathrm{pH} 7.2$, through the splenic artery. Cubic materials, $5 \mathrm{~mm}^{3}$, for each, were dissected, rinsed with the buffer and post-fixed with $1 \%$ osmium tetraoxide in the buffer, two hours at $4^{\circ} \mathrm{C}$. After rinsing with the buffer, they were dehydrated with alcohol, put in iso-amyl acetate, and were frozen with liquid nitrogen and fractured for obtaining fresh surfaces. Then the specimens were treated in a $\mathrm{CO}_{2}$ critical point drier, and were coated with carbon and gold for scanning electron microscopy, SUPER MINISEM and JSM-U3.

Fixed materials were cut at $3 \mu \mathrm{m}$ thick and stained with hematoxylin-eosin, Azan's and Weigert's solution.
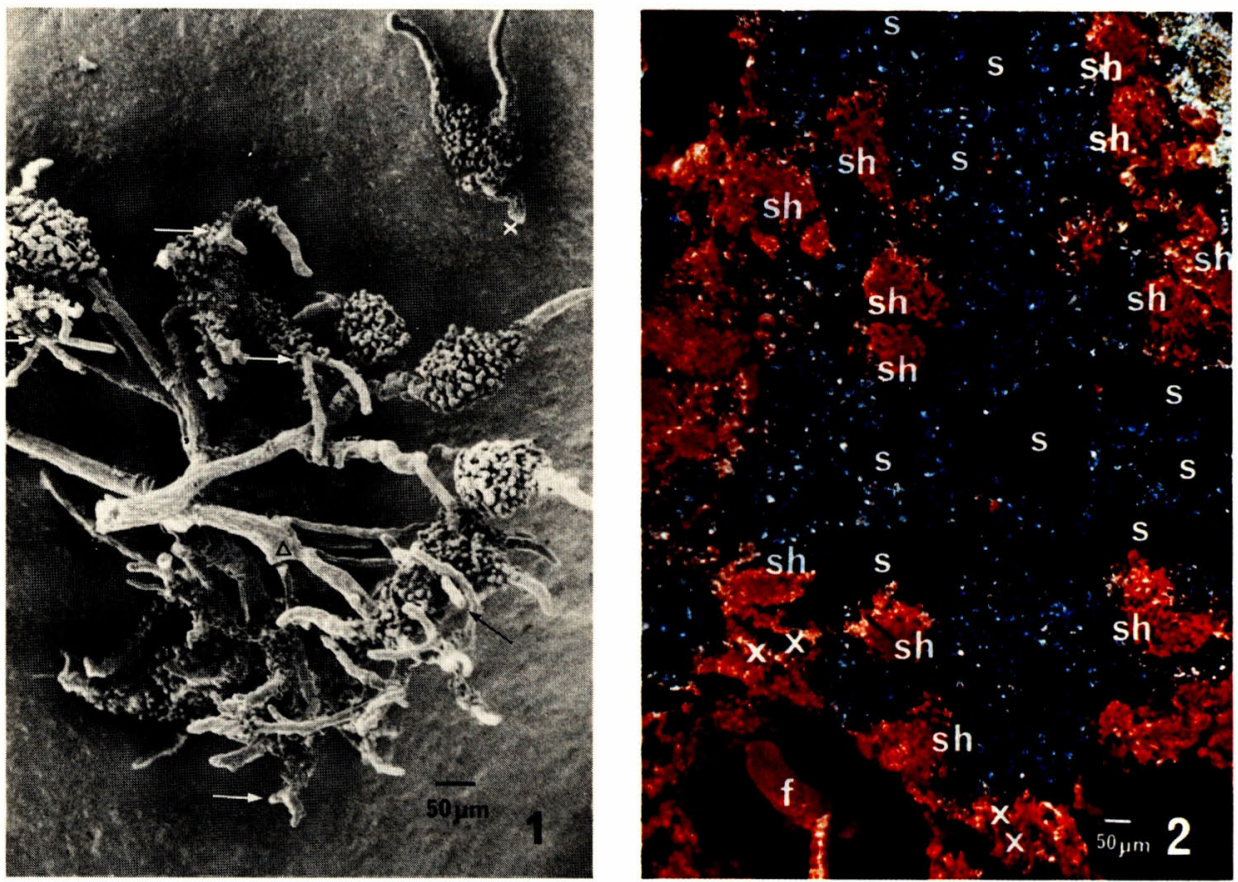

Fig. 1. Ramification of the so-called penicillar artery. It branches into five, and each of them bears one sheath. Thirteen sheathes are clearly observed, but other sheathes lie behind. Some of the arteries bifurcate $(\rightarrow)$ immediately after passing the sheath. A sheathed central capillary $(x)$ separated from a point $(\Delta)$ bifurcates at the proximal end of this sheath, so it is called a biconated sheath. A triconated sheath is seen ( $\backslash$ ).

Fig. 2. Red-colored plastic was injected into the splenic artery and blue-colored plastic into the splenic vein. Sheathes are indicated by sh. In the lower side, a thick follicular artery (f) and the surrounding red pulp are seen. This limited space of the red pulp is easily filled with red plastic injected into the arterial side and connects with the reticular space outside $(x)$, that is, indicated with a faded or mixed-colored plastic. Real blue area is the splenic venous system (s). 


\section{Observations}

The observation has been performed mainly on the corrosion specimen of the sheath and its surrounding structures, while a constant endeavor has been made to substantiate the findings of the corrosions by those observed parenchymal specimens, such as light microscopic histological slides and scanning electron microscopic specimens.

1. Basic form of the pig sheath

Individual sheath appears to be fusiform, pointed at its distal end, that is, similar to a type of projectile (Figs. 1, 2). Size of it is $36 \sim 84 \mu \mathrm{m}$ wide at the proximal side, $83 \sim 216 \mu \mathrm{m}$ long. Also in many cases, sheaths are biconated or rarely triconated as two or three sheaths adhere to one another near their proximal ends (Fig. 1). In these cases, actually the sheathed capillary bifurcates (Fig. 7) or trifurcates within a sheath. The termination of the postsheathed vessel pours into the reticular space of the red pulp (Fig. 7). The diameter of a sheath on a cross section of it is largest at one third of the proximal side, then decreases toward the distal end.

2. The wall of the sheathed central capillary

Different features (Fig. 6) are seen on lumina of the presheathed vessel, the sheathed vessel and the postsheathed vessel, respectively. The diameter of the presheathed vessel is similar to that of the postsheathed, or slightly larger. The postsheathed vessel very often bifurcates at the distal end of the sheath (Figs. 1, $6 \&$ 7). The diameter of the sheathed vessel becomes suddenly smaller (Fig. 6), so small that the vessel has been called the sheathed central "capillary" by many scholars. The cross section of the sheathed capillary appears to be round but irregularly indented (Figs. 3, 4, 8).

On the luminal surface of the sheathed capillary there are three to five distinct longitudinal retractions (Figs. $3,4,8$ ). So the cast surface of the capillary has longitudinal ridges corresponding to the retractions of the luminal surface (Fig. 5). Areas between the retractions on the luminal surface, that is, between the longitudinal ridges on the cast surface, project into the lumen inside (Figs. 3,4) and are composed of endothelial cells (Figs. 8, 9). Along the bottom of each retraction, a row of stigmata (Fig. 5), about $3 \mu \mathrm{m}$ in diameter, are seen at almost regular intervals of about $12 \mu \mathrm{m}$ between stigmata. Such retractions and stigmata are unable to observe in the luminal surface of ordinary arteries in other organs. It is very difficult to catch erythrocytes which are just emigrating through fenestrations of the sheathed capillary wall in every specimen. But, an erythrocyte just located on the fenestrations is observed in a few of specimens (Figs. 8, 9). In a longitudinally sec- 


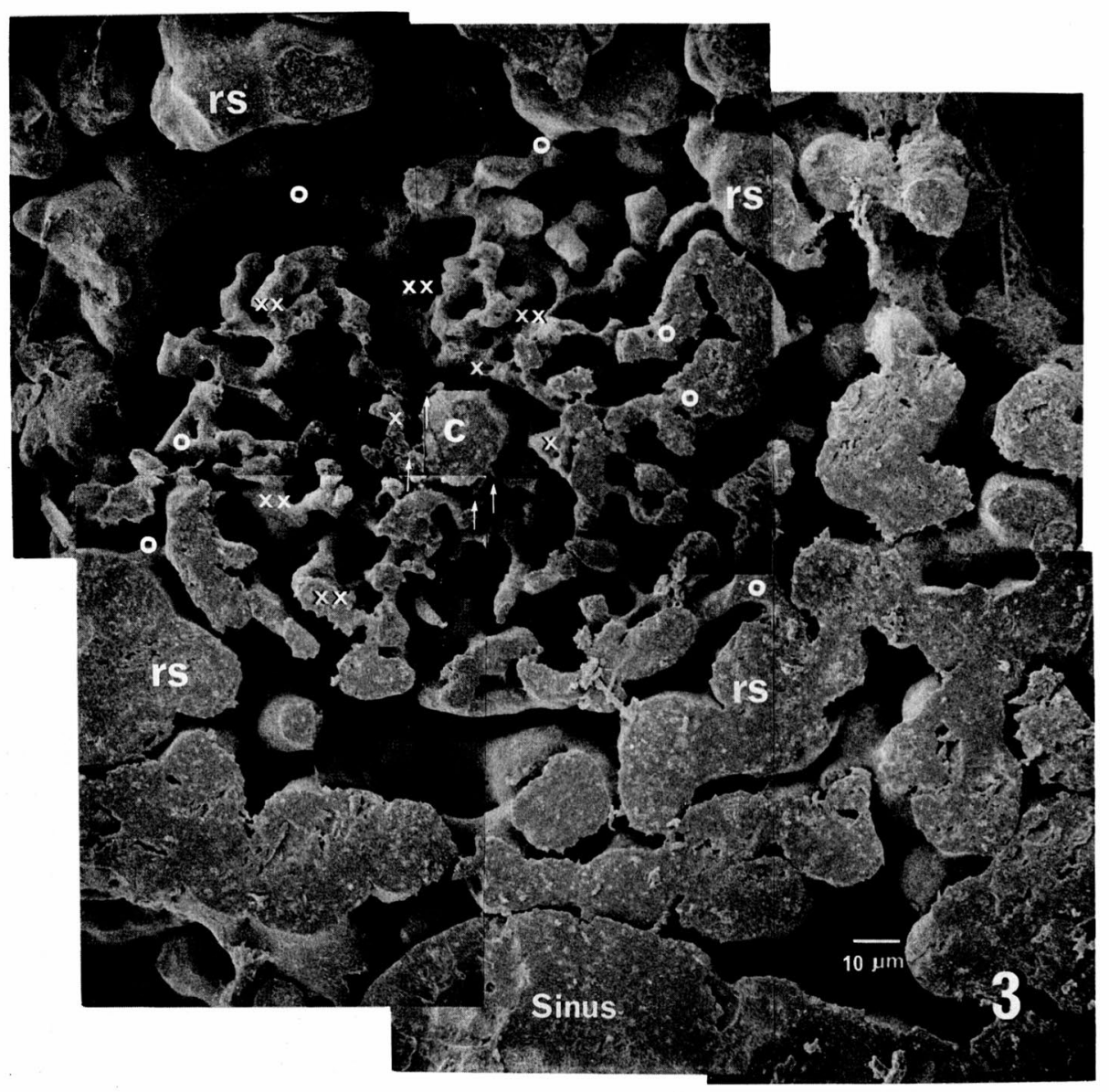

Fig. 3. Cross section of the sheath including the surrounding red pulp and the splenic sinus. A sheathed central capilly (c) has six retractions. These retractions in this cutting level have four fenestrations $(\uparrow)$, and each of them drains into the passageway within a sheath. Each of the passageways mostly communicates with adjacent ones in their beginning $(x)$, and radiates gradually toward the outside of the sheath making anastomoses with one another. Immediately inside the limiting membrane of the sheath, they again form an incomplete circular communication $(x x)$. The reticular network or its component of the periglumaous reticular space does not appear an equal width in a circle, but it shows pretty wide at the upper and lower areas especially in this specimen. Communications between the passageways and the periglumaous space are clearly observed in seven points (O). Then, some of them connect with the reticular space of the red pulp ( $r s$ ). 
tioned sheath, the injected plastic fills the lumen of the sheathed capillary, then penetrates through the fenestrations into the sheath (Fig. 5). Each of the fenestrations appears as round (Fig. 8) or often somewhat elongated along the longitudinal axis of the sheath and is $3 \sim 5 \mu \mathrm{m}$ in diameter. The distribution of the fenestrations is denser in the proximal and the middle areas of the sheathed capillary where its diameter is larger, and becomes sparser toward the distal end of it (Figs. 6, 7).

3. The passageway within the sheath

The injected plastic which leaked through the fenestrations of the sheathed capillary wall proceeds in passageways radiating in all directions within the sheath (Figs. 3,4). The diameter of each passageway is $2 \sim 4 \mu \mathrm{m}$, and it usually takes a little zigzag course between cellular elements of the sheath. This is called the intrasheathed passageway by the present authors. When the passageway radiates toward the outer surrounding of the sheath, it communicates with adjacent ones, as well with both proximal and distal radiating passageways (Fig. 3). Eventually these communications within one sheath develop into a projectile which is formed by a three dimensional mesh work (Fig. 6). The course of passageway appears to be irregular and without any definite network when a single sheath is examined. The authors, however, have been able to demonstrate an arrangement between the course and form of the passageway when the observations are made carefully on numerous
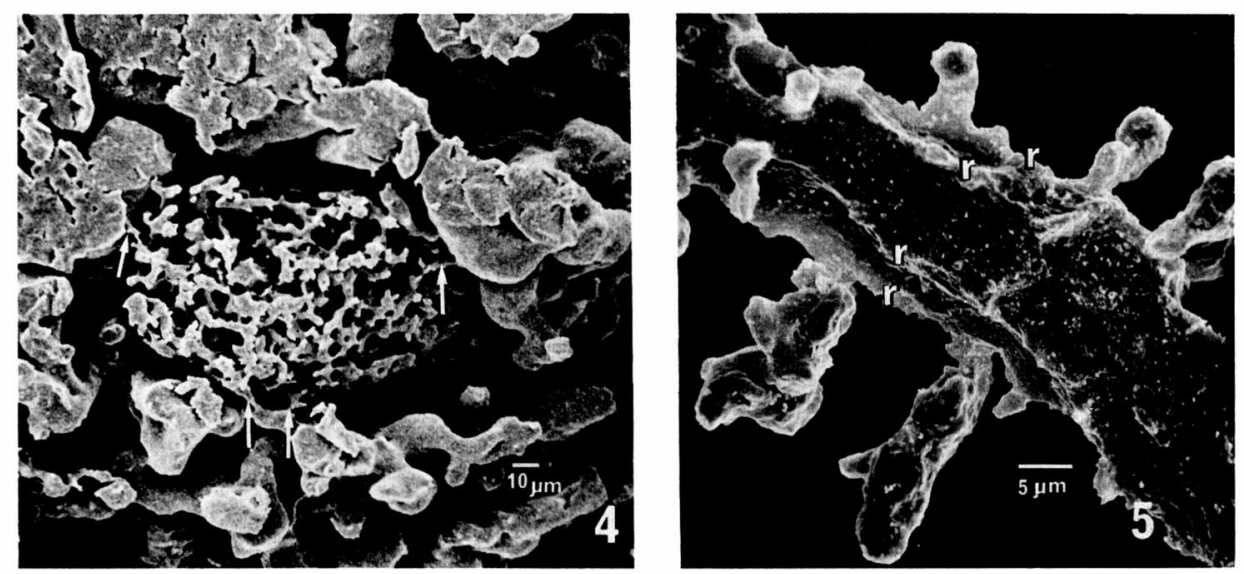

Fig. 4. This corrosion specimen was prepared by the injection into the fixed spleen. As has been shown in Figure 3 , the retractions on the luminal surface, the passageways and four communications $(\uparrow)$ between the passageways and the periglumaous space, are seen.

Fig. 5. Four longitudinal ridges $(r)$ are seen on this side of the cast of the sheathed central capillary. The plastic material flows from the fenestrations which are located holding almost regular intervals between them on the ridges. 
casts of the sheaths of even other species of mammals, as mentioned above. The diameter of the passageway would allow a single erythrocyte just to pass through. Erythrocytes, though most of them are deformated, observed in SEM specimens (Figs. 8, 9), are held tight between sheathed cells which surround the passageway, the actual component of the wall of it. Form of the passageway is narrower and delicate, but just prior to reaching the limiting fibrous membrane the passageway becomes enlarged, forming small balloon-like terminals (Fig. 3).

4. Communication between peripheries of the passageway and the red pulp

The enlarged spaces, the terminal ends of the radiating passageways connect with each other inside the limiting fibrous membrane (Figs. 3, 7) where there is a denser network of the reticular fibers. These terminals are seen passing through the limiting membrane in only a few area (Fig. 3), not all around the periglumaous areas. Then, they connect

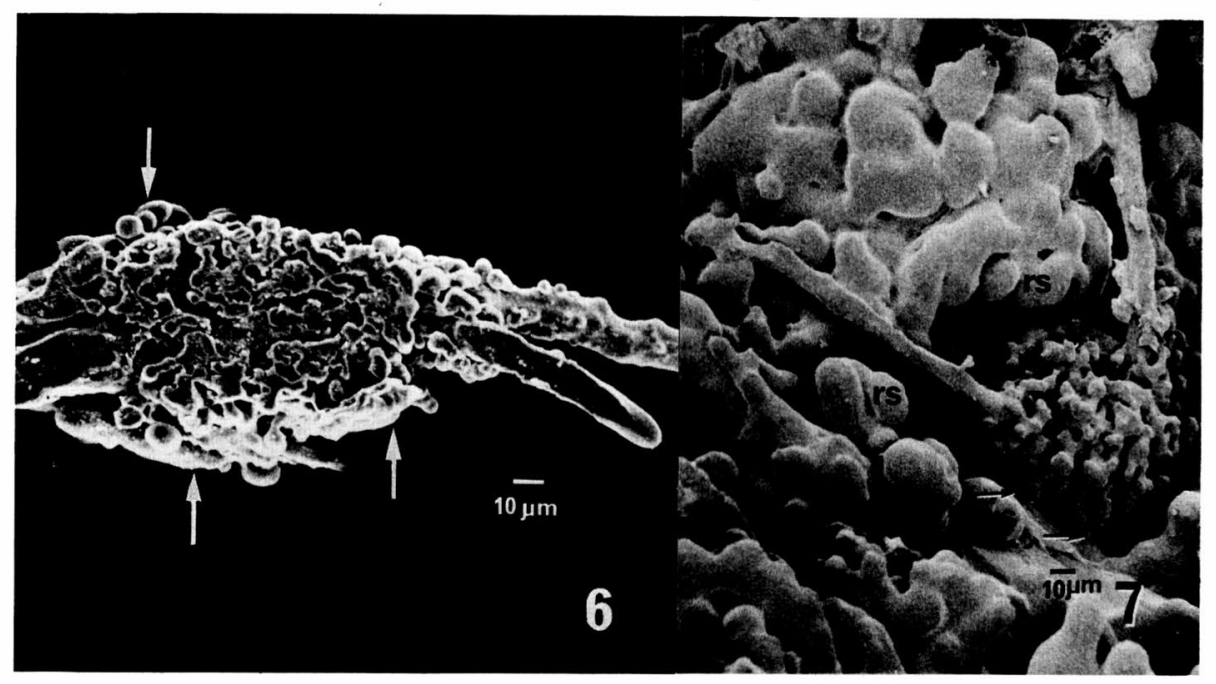

Fig. 6. The cast of a sheath sectioned longitudinally. The diameter is largest in the presheathed vessel, smallest in the sheathed vessel and larger in the postsheathed vessel. The distal end of the postsheathed vessel bifurcates. The passageways extend not only in the perpendicular direction of the sheath, but also make communications in the longitudinal direction. Portions indicated by arrows are the periglumaous space.

Fig. 7. The distal end of the sheath and the postsheathed vessels. Cast of the reticular space of the red pulp between two vessels is removed. The passageways of the sheath connect (arrows) with the periglumaous space. Area of the limiting fibrous membrane of the sheath is shown as a space surrounding the sheath. Both termini of the two postsheathed vessels pour into the reticular space of the red pulp. 
(Figs. 3, 4\& 7) with the periglumaous space which is part of Billroth's reticular red pulp space; in other words, continue through the limiting membrane in a limited number of areas. Therefore, the passageways within the sheath are quite different and very distinct from the spaces observed in the red pulp (Figs. 4, $6 \& 7$ ).

\section{Discussion}

Although it has been thoroughly discussed that the sheathed central vessel is called either an artery or a capillary by employing various light microscopic approaches, it does not seem to be concluded yet.

The sheath was considered to be an active contractile muscular organon by some classic workers, but such a view has not been regarded at present. While, Solnitzky (1937) stated that the sheathed vessel did not belong to an arterial vessel because an experimentally injected pigment leaked from its wall. Some scholars who studied the splenic construction of the argyrophil fiber, have mentioned that the sheath was made of a thickened reticular network and did not contain any muscular fibrous element. Ono (1930), Imai and their coworkers (1940, 1947, 1954) maintained their opinion that the sheath was fusiform swelling of a limited area of the pulpal arterial channel which was derived from the adventitia accessoria of the embryonic arterial vessel.

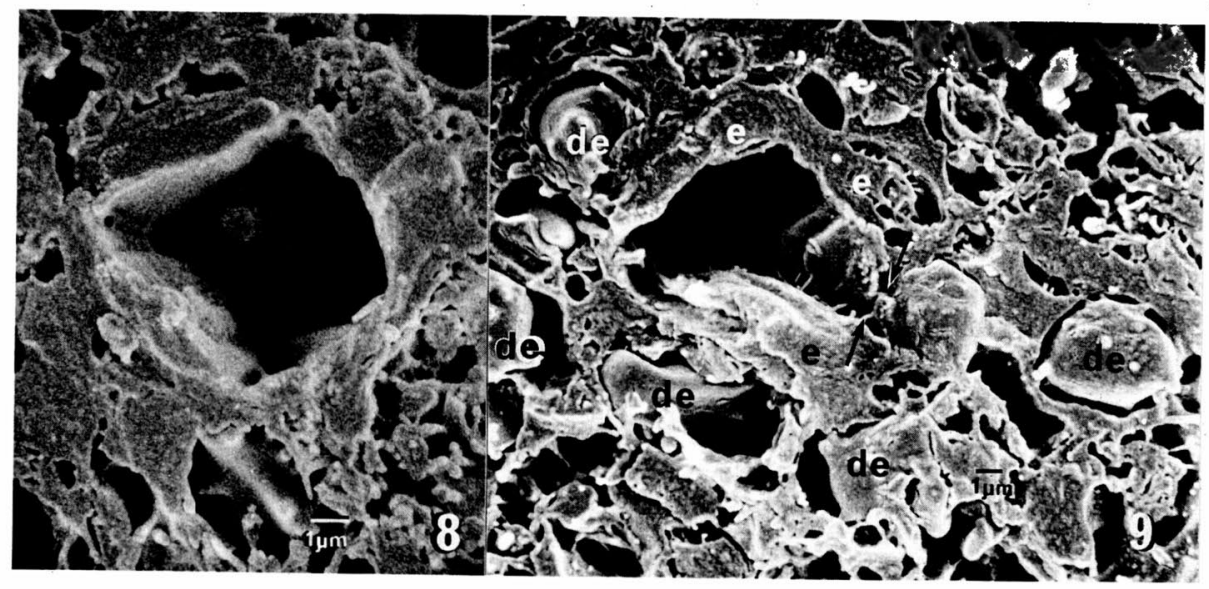

Fig. 8. The cross section of the sheathed vessel in the narrowest lumen of it. Three endothelial cells are seen, and the longitudinal retractions are located between them. Three retractions in Figure 7 bear the fenestrations, from one of which in the deeper area a deformed erythrocyte is emigrating. A deformed erythrocyte in the bottom is located immediately adjacent to the endothelial cells.

Fig. 9. Cross section of the sheathed central capillary. A deformed erythrocyte is emigrating through a fenestration indicated by two arrows. Endothelial cells (e). Deformed erythrocytes (de) are located tightly outside the endothelial cells and between sheathed cells. 
So this area was an undifferentiated, primitive, arterial capillary without any arterial function. They finally have concluded the sheathed vessel was a capillary. Ohta (1957), one of the coworkers of the present paper, made a consideration in his investigation of the comparative study of some mammalian splenic vasculature as follows: The sheathed vessel was extremely small in diameter; retractions were seen on its luminal surface as has been reconfirmed in the present paper; there were a very few of primitive contractile element or not; the pigment injected easily leaked from its wall into the sheath in the pig and cat. Then, he thought it to be reasonable that the sheathed vessel was a capillary. Based on the findings of this paper, the sheath could not but have a filtrating function. Schweiger-Seidel once suggested the sheath was a filtrating apparatus. Many investigators have followed his theory ; some of them mentioned there might be preformed stigmata on the luminal surface of the sheath, and others mentioned no preformed stigmata but that only the liquid vehicle of the blood could extravasate from the wall in certain physiological conditions. A few of them have believed more or less formed blood elements might flow out with the liquid vehicle. Ohta described that the injected acryl plastic leaked from some spots of the sheathed capillary wall under the light microscope and spread in all directions toward the periphery of the sheath. And he made a suggestion of a definite passageway within the sheath. This kind of the passageway could not be demonstrated by color-matter suspensions of water soluble liquids.

Unexpectedly Loreti and Voglioli in the same year reported such findings on the pig sheath by means of the india ink injection. However, these findings of Ohta and Loreti did not progress beyond the light microscopic order. Formerly, Müller (1865) and Banwarth (1891) stated that the appearance of the injected materials in the sheath was no more than an artificial extravasation. But Ohta proved an argument against their statement by some experimental morphological works as follows: The plastic injection was performed into the splenic artery of the dog which was injected the india ink intravenously before sacrifice, and he made an observation that the india ink was found in the sheath, where simultaneously the injected plastic was injected through the sheathed capillary wall at a certain time after the intravenous injection, as well proceeding to the periglumaous space afterward. He after all proved the india ink of the vital injections coexisted with the plastic injected in and around the sheath. The fenestrations on the luminal surface of the sheathed capillary were confirmed under the scanning electron microscope. It might mean that the fenestration was preexistant in a certain physiological condition. Furthermore the passageway within the sheath was observed on the fixed specimens before the plastic injection (Fig. 4). Based on these findings, the authors be- 
lieved that the fenestration and the passageway were not artifact which was produced by injection material or pressure.

The lateral branches of the sheathed capillary which were described by Snook (1950) and Ohtsuka (1955) were no more than the passageway in itself demonstrated in the present paper. In other words, the capillary of the pig did not diverge such a lateral branch. The cast of the passageway was quite different from that of the reticular space of the red pulp surrounding the sheath in the form, size and mutual communi cations.

The present authors have come to a consideration about the circulating route of the erythrocytes in the sheath as follows: Erythrocytes first emigrate through the fenestration and proceed at much slower pace through the definite passageway up to just inside the limiting fibrous membrane. Then, they flow into the periglumaous space through the limited area of the membrane. Furthermore, after the spleen was washed by Ringer's solution through the splenic artery, it was observed that many erythrocytes still were left between the sheath cells. Judging from this fact the erythrocytes have not proceeded only much slowly, but many of them would be obliged to stay between the sheathed cells which formed narrow passageways, where the erythrocytes must be affected by the slower passing or staying of the blood flow.

\section{Summary}

Passageways within the splenic sheath of the pig was studied by means of the plastic injection method. On the luminal wall of the sheathed central vessel, the longitudinal retractions were seen, and a row of stigmata left each bottom of the retractions in almost regular intervals. The fenestration continued as a passageway which radiated in all directions within the sheath. The passageways communicated with one another, but morphologically different from the reticular space of the red pulp. Each terminal of the passageways became enlarged, and anastomosed with adjacent ones inside the limiting fibrous membrane. Then, they communicated with the periglumaous space, not all around the sheath but in a few area. These findings were obtained on the injected materials into the splenic artery and vein in both fresh and fixed spleens.

\section{References}

1) Bannwarth, J.: Untersuchngen über die Milz. Arch. f. mikr. Anat., 38 : 345-446, 1891.

2) Imai, T.: Function and structure of the ellipsoid of the cat's spleen. Fukuoka Acta med., 33 : 960-970, 1940 (in Japanese). 
3) Imai, T.: Reticular framework of the Schweigger-Seidel sheath of the spleen. Fukuoka Acta med., 38 : 1-5, 1947 (in Japanese).

4) Imai, T.: Problems on the vascular ellipsoid of the spleen, with special reference to its morphological findings. Symposium on hematology. $7: 1-86,176-202,1954$ (in Japanese).

5) Loreti, F., e C. Vogliotti : Identificazione plastica per corrosione dei manicotti lienali pericapilleri (Hülsenkapillaren) in sus scrofa. Z. Zellforsch., 46 : 345-346, 1957.

6) Müller, W.: Über den feineren Bau der Milz. Leipzig-Heidelberg, 1865.

7) Ohtsuka, Y.: Relationship between histological appearance of the SchweiggerSeidel sheath and the general structure of the spleen. J. Kyu. Soc., 5 : 104-210, 1955 (in Japanese).

8) Ohta, Y.: Cubical anatomy of several ducts and vessels by injection method of acrylic resin. III. On the vascular system of the spleen in some mammals. Okajimas Fol. anat. jap., $30: 13-41,1957$.

9) Ohta, Y., H. Hanai, H. Sawa and T. Fujimoto: Cubical anatomy of several ducts and vessels by injection method of acrylic resin. IV. On the vascular system of the spleen in rat and mouse. Okajimas Fol. anat. jap., $31: 407-417,1958$.

10) Ono, K.: Untersuchungen über die Entwicklung des menschlichen Milz. Z. Zellforsch. mikr. Anat., 10:573-603, 1930.

11) Schweigger-Seidel, F.: Untersuchung über die Milz. Arch. path. Anat. u. Physiol., 27 : 460-504, 1863.

12) Snook, T.: A comparative study of the vascular arrangements in mammalian spleens. Am. J. Anat., 87: 31-78, 1950.

13) Solnitzky, O.: The Schweigger-Seidel sheath (ellipsoid) of the spleen. Anat. Rec., 69 : $55-70,1937$.

14) Taniguchi, Y., Y. Ohta and S. Tajiri : New improved method for injection of acrylic resin. Okajimas Fol. anat. jap., 24 : 259-267, 1952.

15) Taniguchi, Y., Y. Ohta, S. Tajiri, H. Okano and H. Hanai.: Supplement to new improved method for injection of acrylic resin. Okajimas Fol. anat. jap., $27: 401$ 406, 1955.

16) Tischendorf, F.: Die Milz. Handbuch d. mikr. Anat. d. Mensch. von W. Möllendorff, Sechster Teil, Springer-Verlag, Berlin, 1969. 